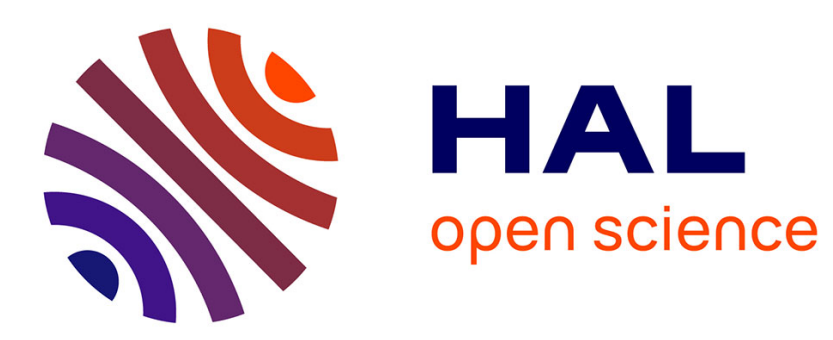

\title{
Communication verbale et insertion professionnelle: analyse de la construction collaborative du discours chez les locuteurs natifs faiblement qualifiés
}

Valérie Langbach

\section{- To cite this version:}

Valérie Langbach. Communication verbale et insertion professionnelle: analyse de la construction collaborative du discours chez les locuteurs natifs faiblement qualifiés. Revue Française de Linguistique Appliquée, 2014, XIX (2), pp.85-95. 10.3917/rfla.192.0085 . hal-01107805

\section{HAL Id: hal-01107805 \\ https://hal.science/hal-01107805}

Submitted on 5 Dec 2019

HAL is a multi-disciplinary open access archive for the deposit and dissemination of scientific research documents, whether they are published or not. The documents may come from teaching and research institutions in France or abroad, or from public or private research centers.
L'archive ouverte pluridisciplinaire HAL, est destinée au dépôt et à la diffusion de documents scientifiques de niveau recherche, publiés ou non, émanant des établissements d'enseignement et de recherche français ou étrangers, des laboratoires publics ou privés. 


\title{
Communication verbale et insertion professionnelle : Analyse de la construction collaborative du discours chez les locuteurs natifs faiblement qualifiés
}

\author{
Langbach, Valérie \\ ATILF \\ Université de Lorraine \& CNRS \\ Valerie.langbach@univ-lorraine.fr
}

\section{Introduction}

Dans un monde du travail où les compétences nécessaires à l'exercice d'une profession s'accroissent et se diversifient, où le statut de demandeur d'emploi se professionnalise (Divay, 2011), les pratiques langagières des demandeurs d'emploi nous semblent emblématiques des nouveaux défis rencontrés par les politiques d'insertion. L'objectif de notre analyse est de dégager les caractéristiques qui sous-tendent le fonctionnement des interactions verbales dans les situations d'entretien d'insertion pour vérifier ensuite si les locuteurs-demandeurs d'emploi faiblement qualifiés sont aptes à entrer dans une situation de communication satisfaisante dans ce cadre communicationnel, c'est-à-dire «à entrer en communication de façon efficace et de se trouver en phase sociolinguistique avec un maximum de locuteurs différents dans le plus grand nombre possible de situations de communication » (Adami, 2007 p.35). Dans un premier temps, nous présenterons le cadre dans lequel s'inscrit cette étude afin de préciser notre approche sociolinguistique des interactions verbales. Dans un second temps nous préciserons les stratégies de gestion de l'interaction mises en œuvre dans la construction collaborative du discours et notamment, un phénomène discursif récurent : la reprise. Nous présenterons ensuite le corpus de travail utilisé pour cette étude ainsi que la méthodologie de recueil et d'analyse de celle-ci. Enfin, dans la quatrième partie de cet article, nous analyserons des exemples de gestion de l'interaction verbale qui feront apparaitre à la fois les stratégies mises en œuvre par le locuteur qualifié et le locuteur non qualifié pour co-construire un échange satisfaisant. Notre analyse s'est focalisée sur le rôle du locuteur qualifié dans la construction collaborative du discours et sa gestion du malentendu, notamment à travers le phénomène de reprise. 


\section{Sociolinguistique des interactions verbales}

Notre analyse de la construction collaborative du discours, et plus précisément dans le cadre de cette étude, de la construction collaborative du discours entre un locuteur qualifié et un locuteur natif faiblement qualifié en situation d'insertion professionnelle, nous amène à faire appel à une approche sociolinguistique des interactions verbales (André, 2010).

Les interactions se caractérisent par une adaptation et des ajustements permanents des locuteurs engagés conjointement dans la construction collective de leurs discours (Schegloff, 1982), et en fonction des différents éléments de la situation de communication (Hymes, 1972), notamment par l'identité, le statut et le rôle conversationnel des interlocuteurs (Goffman, 1981). L'interaction verbale est située, contextualisée. Kerbrat-Orecchioni (1996, p.4-5) ajoute :

Pour qu'il y ait échange communicatif, il ne suffit pas que deux locuteurs (ou plus) parlent alternativement ; encore faut-il qu'ils se parlent, c'est-à-dire qu'ils soient tous deux 'engagés' dans l'échange, et qu'ils produisent des signes de cet engagement mutuel, en recourant à divers procédés de validation interlocutoire.

Dès lors que les interactants s'engagent mutuellement, dans une situation de face à face, ils collaborent et co-construisent le discours (André, 2014). Kerbrat-Orecchioni (2000), précise que ces influences mutuelles peuvent être plus ou moins fortes en fonction de la nature de la situation de communicative rencontrée. Pour rendre compte de l'ensemble des facteurs influençant la production et le déroulement des interactions verbales dans le cadre spécifique des entretiens d'insertion notre approche se doit d'être pluridisciplinaire et multidimensionnelle. Nous avons construit un cadre analytique des interactions verbales dans lequel nous avons puisé les concepts, issus notamment de la linguistique interactionnelle (Kerbrat-Orecchioni, 2005), de l'analyse conversationnelle (Sacks, Schegloff et Jefferson, 1974 ; Schegloff, 1982), des maximes conversationnelles de Grice (1979) adaptées à l'analyse des interactions qui étaient nécessaires à l'analyse des données recueillies sur le terrain. Enfin nous avons réservé une place conséquente à l'analyse du phénomène de reprise (André, 2010 ; Vion, 1992-2005) véritable fil rouge de notre étude.

\section{Les stratégies de gestion de l'interaction}


Pour décrire les différentes stratégies de communication mises en œuvre tant par le locuteurconseiller, qui mène l'entretien d'insertion, que par le locuteur-demandeur d'emploi pour négocier le sens de l'échange et tendre vers une communication satisfaisante, nous avons repris les modèles de Faerch et Kasper (1980) et de Giacomi et de Heredia (1986), qui étudient les différents types de stratégies mises en œuvre par les locuteurs non natifs pour communiquer. Faerch et Kasper (1980) proposent trois types de stratégies que nous précisons ci-dessous :

\section{LES TROIS TYPES DE STRATEGIES}

Synthèse des travaux de Faerch et Kasper (1980)

\begin{tabular}{cc}
\hline $\begin{array}{c}\text { Les stratégies de } \\
\text { réduction } \\
\text { formelle }\end{array}$ & $\begin{array}{c}\text { "[l'apprenant] communique à l'aide d'un système réduit, afin d'éviter de } \\
\text { produire des énoncés laborieux ou incorrects du fait de règles ou items } \\
\text { insuffisament automatisés ou hypothétiques." }\end{array}$ \\
\hline $\begin{array}{c}\text { Les stratégies de } \\
\text { réduction } \\
\text { fonctionnelle }\end{array}$ & "[l'apprenant] réduit ses objéctifs communicationnels afin d'éviter un \\
problème."
\end{tabular}

Les travaux de Giacomi et de Heredia (1986), quant à eux, distinguent deux catégories de stratégie de communication : les stratégies préventives et les stratégies de gestion.

- Les stratégies de prévention servent à anticiper et ainsi éviter les situations d'incompréhension qui peuvent naitre dans les situations exolingues. Pour les auteurs (1986, p.16-17), ces stratégies renvoient à « toutes tentatives d'ajustement faites par les locuteurs - et particulièrement natif, mais pas seulement celui-ci - pour rendre leur discours au maximum compréhensif ».

- Les stratégies de gestion renvoient à l'ensemble des procédures de gestion auxquelles ont recours les locuteurs pour tenter de résoudre un problème de communication en vue de la poursuite immédiate des échanges. La mise en œuvre de ces stratégies est généralement marquée par l'interruption de l'échange en cours. Un des locuteurs passant de l'échange 
informatif pour entrer dans une activité d'ordre métalinguistique : celui-ci, par des questions de clarifications, vérifie la compréhension des propos émis ou demande des éclaircissements, ce que les auteurs (Ibid., p.20) décrivent comme «une phase de négociation parenthétique, nommée ainsi parce qu'elle constitue une sorte de parenthèse par rapport à l'échange en cours ».

Pour Giacomi et de Heredia (Ibid.), ces stratégies révèlent une tentative des locuteurs d'améliorer une communication devenue problématique pour réorienter l'échange vers une communication satisfaisante.

L'étude de ces différentes stratégies nous amène à analyser plus finement une forme discursive récurrente utilisée tant par le locuteur qualifié que par le locuteur non qualifié pour co-construire le discours : la reprise (André, 2010; Vion, 1992; 2005) Différentes classifications des phénomènes de reprise (voir notamment la classification De Gaulmyn (1987, p.87) ou encore la typologie de Gülich et Kotschi (1987)) renvoient à une multitude de termes : reprise, reformulation, ou encore répétition. Pour analyser les phénomènes rencontrés dans notre étude, nous adopterons une définition générique de la reprise. D’un point de vue interactionnel et à la suite de Vion (1992), nous définirons la reprise comme « le fait de s'approprier certains aspects de la parole d'autrui en les incorporant dans son propre discours. Nous devons distinguer cependant deux types de reprise (Roulet, 1985 ; Vion, 1992) : les auto-reprises ou réitération partielle ou intégrale du discours du locuteur par lui-même et les reprises diaphoniques ${ }^{l}$ ou insertion du discours d'un autre locuteur dans son propre discours. Vion (Ibid., p.215), complètera cette définition en mentionnant les reprises réitératives qui se contentent de reprendre le discours initial et la reprise codée « dans lesquelles l'acte de reprise est explicité par des formes linguistiques du type quand je dis... ou quand vous dites ... ».

Nous ajouterons à la suite de Ducrot (1980, p.7), que si la reprise est «immédiate et à l'identique d'un fragment discursif par un même locuteur [elle] ne doit pas nous faire oublier, que tout énoncé constitue un événement singulier ». C'est pourquoi nous nous attacherons à analyser chacune de ces formes rencontrées dans notre corpus comme un nouveau tour de parole participant à la construction collaborative du discours, porteur d'une valeur et une signification singulière en référence au segment discursif antérieur. Dans le cadre de cette

\footnotetext{
${ }^{1}$ Par opposition à l'auto-reprise, nous utiliserons également le terme de « hétéro-reprise ».
} 
étude, nous nous intéresserons plus particulièrement à la reprise diaphonique réitérative, forme récurrente dans notre étude.

\section{Corpus de travail et méthodologie d'analyse des données}

L'ensemble de cette recherche s'est déroulé dans les locaux de l'organisme de formation, Formabilis, Association loi 1901, dans lequel nous sommes salariée depuis quinze années. Cet organisme est prestataire Pôle Emploi, c'est-à-dire qu'il effectue, pour Pôle Emploi, des prestations d'accompagnement auprès de demandeurs d'emploi. Pour recueillir les données nécessaires de notre étude, nous avons utilisé l'entretien. Le corpus de travail utilisé dans le cadre de cette étude est composé de 49 entretiens semi directifs : 39 demandeurs d'emploi et 4 professionnels de l'insertion. La durée totale de notre corpus est de $10 \mathrm{~h} 05 \mathrm{mn} 58 \mathrm{~s}$. Il est décomposé en 6 sous corpus, en fonction des types d'entretiens. Si la majorité de notre corpus est composée d'entretiens avec des locuteurs-demandeurs d'emploi faiblement qualifiés (infra niveau V) - population cible de notre recherche - nous avons choisi d'intégrer à notre étude quelques entretiens avec des locuteurs qualifiés (de niveaux $\mathrm{BAC}+2$ à $\mathrm{BAC}+5$ ).

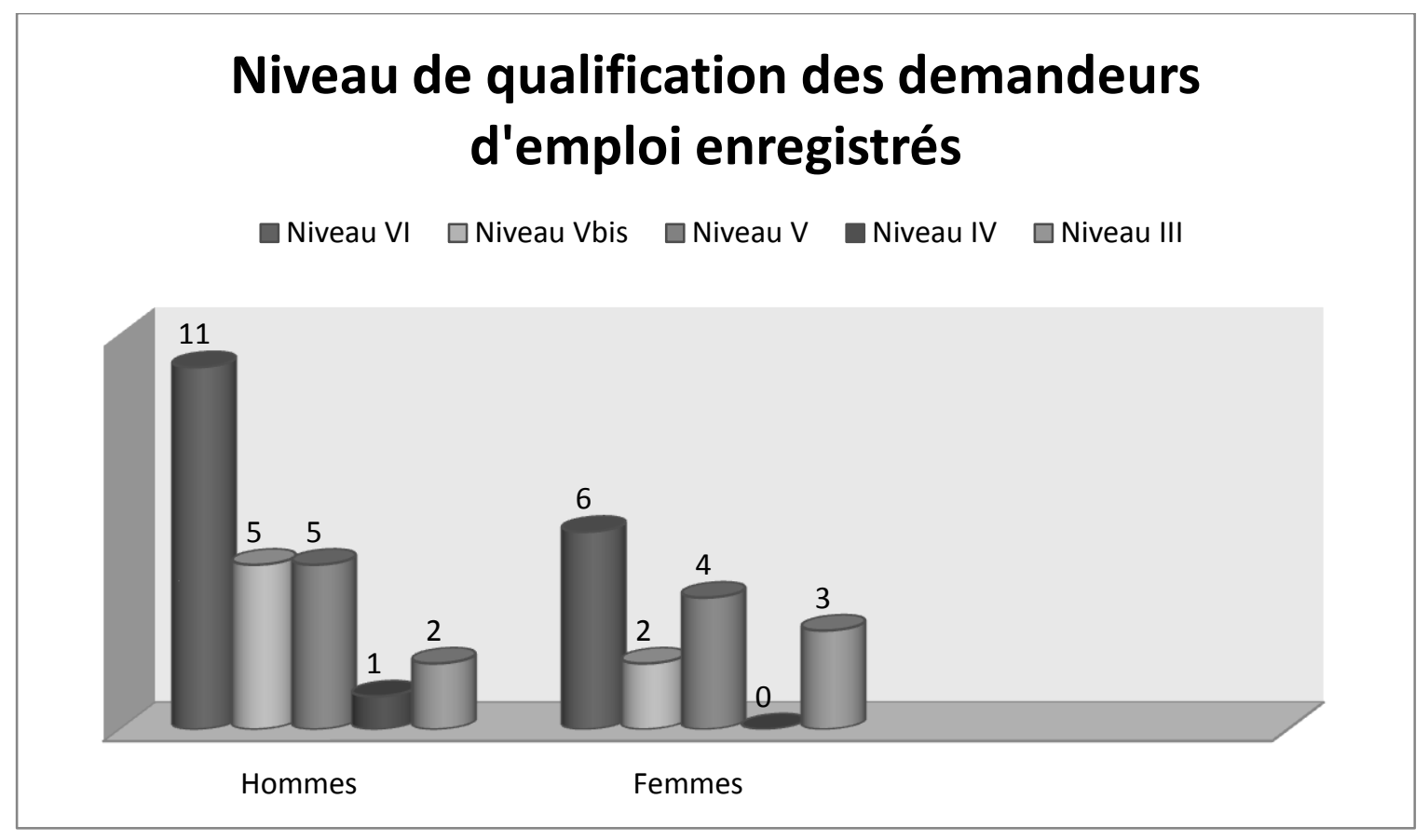

Pour le sous corpus 01, constitué de 4 entretiens, nous sommes intervenue en tant que conseiller dans un premier temps, puis comme chercheur. Nous avons opté pour une présentation succincte de notre recherche évoquant simplement le cadre disciplinaire (les 
sciences du langage) et l'enjeu «global» de celle-ci : «faire une étude sur la façon dont les individus parlent de leur parcours professionnel».

Dans le sous corpus 02 (constitué de 12 entretiens), nous sommes intervenue en tant que conseiller lors d'atelier Pôle Emploi.

Pour les sous corpus 03 (10 entretiens), 04 (4 entretiens), nous avons été avant tout présentée comme: un collègue qui effectue une enquête pour améliorer les pratiques d'accompagnement (sous corpus 03) ou de formation (sous corpus 04) des personnes en situation d'insertion accueillies dans l'organisme.

Le sous corpus 05 (10 entretiens) a été enregistré par un conseiller collègue lors de ses séances d'accompagnement avec des demandeurs d'emploi.

Enfin, pour le sous corpus 06 (4 entretiens), nous sommes intervenue en tant que chercheur lors d'entretien avec des collègues conseillers.

L'ensemble des données orales de notre corpus a été enregistré et transcrit. Nous avons utilisé la convention de transcription mise au point par l'équipe du projet TCOF (Traitement de Corpus Oraux en Français) de l'ATILF ${ }^{2}$. Les principes généraux retenus pour transcrire notre corpus et en lien avec les objectifs de notre recherche sont notamment de transcrire tous les propos des interlocuteurs, y compris les hésitations et les répétitions selon les règles de l'orthographe usuelle, sans ponctuer les énoncés. Nous avons effectué l'ensemble des transcriptions. La proximité qui s'est construite avec notre corpus tout au long de la transcription, nous a permis d'établir très rapidement des liens entre différentes structures langagières récurrentes ou de vérifier d'autres hypothèses de travail. Notre méthodologie d'analyse s'est déroulée en deux temps. Dans un premier temps, à travers une approche sociolinguistique des interactions, nous avons relevé les spécificités et les implications dans l'interaction verbale du genre spécifique d'échange que nous avons qualifié d'entretien d'insertion professionnelle ou d'accompagnement. En d'autres termes, nous avons tenté de prendre en compte l'ensemble des facteurs extralinguistiques qui influence le déroulement des interactions verbales. Nous avons ensuite effectué une analyse des données qui a fait émerger des formes linguistiques et interactionnelles prépondérantes dans les situations de communication en insertion avec des personnes faiblement qualifiées. Notre analyse s'est focalisée sur le rôle du locuteur-conseiller dans la construction collaborative du discours notamment à travers le phénomène de reprise.

\footnotetext{
${ }^{2}$ www.cnrtl.fr/corpus/tcof
} 


\section{Analyse de la construction collaborative du discours}

A partir de l'analyse de nos différents sous corpus, nous pouvons proposer un modèle de construction collaborative différent en fonction du niveau de qualification du locuteurdemandeur d'emploi. L'interaction verbale entre les interlocuteurs reprend le schéma classique de paires adjacentes : question-réponse (Schegloff, 1973), soit Question de L1 / Réponse de L2 / Ratification de L1. Cependant, ce modèle est plus complexe lors d'une interaction verbale réunissant un locuteur-conseiller et un locuteur-demandeur d'emploi qualifié.

\subsection{Analyse de deux modes de construction collaboratives du discours}

\section{Modèle de la construction collaborative du discours}

Locuteur-conseiller / Locuteur-demandeur d'emploi qualifié

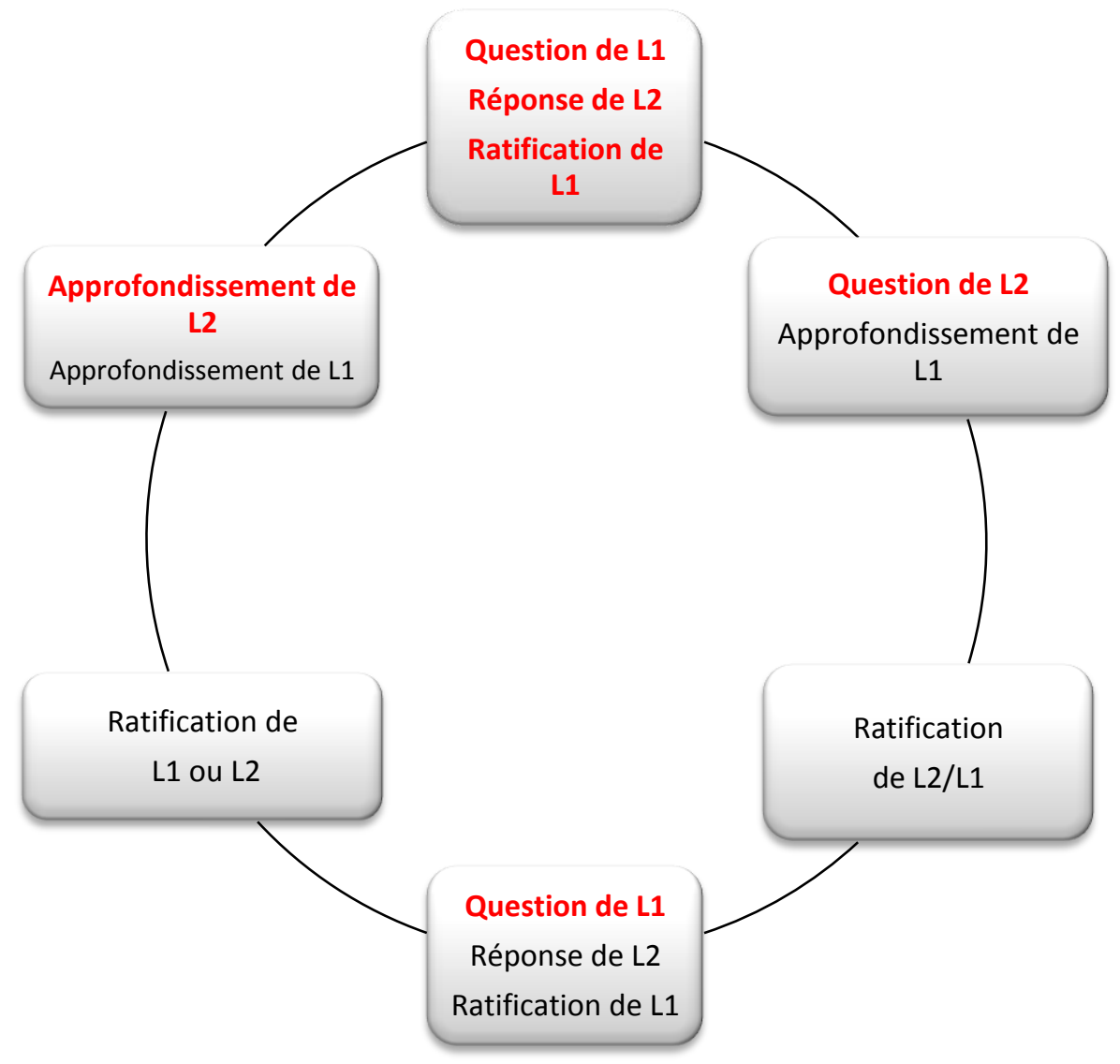


L'exemple ci-dessous illustre ce schéma. A la suite de la ratification du locuteur-conseiller L1, le locuteur-demandeur d'emploi L2 peut, en fonction de la direction qu'il souhaite donner à l'échange, soit poser une question à L1, soit approfondir sa propre réponse. Les interactants peuvent ensuite tour à tour ratifier la réponse donnée par l'autre locuteur, approfondir une nouvelle fois la thématique abordée, et ceci pendant de nombreux tours de paroles jusqu'à l'obtention d'un échange satisfaisant pour les deux parties.

\section{Exemple}

\section{Question L1}

L1 [...] quand vous parlez de variété des tâches c'était quoi par exemple

\section{Réponse L2}

L2 ben par exemple je pense aux demandeurs d'asile ou euh c'était du suivi de dossier c'était aller les voir dans leur chambre d'hôtel les écouter et les soutenir c'était les accompagner à l'école faire des démarches scolaires

\section{Ratification L1}

L1 d'accord

\section{Approfondlissement L2}

L2 enfin c'était du suivi au quotidien euh suivi social et médical parce que j'ai aussi suivi des familles qui avaient une pathologie assez lourde ensuite dans l'enseignement c'est aussi euh variété parce qu'il y a préparation euh préparation des cours organisation du cours puis présentation et intervention à l'oral quoi

\section{Ratification L1}

L1 d'accord sur les compétences développées <polyvalence adaptabilité [Sous corpus 02 / ENR 03 / BAC+5]

Dans cet exemple, le locuteur-conseiller (L1) pose une question au locuteur-demandeur d'emploi (L2). L2 répond à la question de L1. L'information pertinente pour répondre de manière satisfaisante est donnée dans le premier tour de parole «c'était du suivi de dossier et celle-ci est contextualisée je pense aux demandeurs d'asile». L2 propose dans sa réponse quelques informations secondaires pour décrire son activité : aller les voir / les écouter / les soutenir / les accompagner. A ce niveau de l'échange, le contrat de communication est rempli. La première séquence de cet échange est achevée. Le locuteur L1 peut ratifier (ce qu'il fait) et une nouvelle question peut être posée. Cependant, le locuteur L2 approfondit sa réponse parce qu'elle lui semble incomplète «j'ai aussi suivi des familles » mais aussi pour apporter une 
nouvelle information au locuteur L1 concernant sa période professionnelle effectuée dans l'enseignement. Pour clore, L1 ratifie et introduit la thématique suivante «sur les compétences développées <polyvalence adaptabilité ». Une des premières caractéristiques de la collaboration à la construction du discours par les locuteurs-demandeurs d'emploi qualifiés est l'apport d'informations complémentaires.

Contrairement au modèle précédent, la structure de l'échange entre un locuteur-conseiller et un locuteur-demandeur d'emploi faiblement qualifié est moins complexe et présente moins de tours de parole.

Le modèle de construction collaborative du discours

Locuteur-conseiller / Locuteur-demandeur d'emploi faiblement qualifié

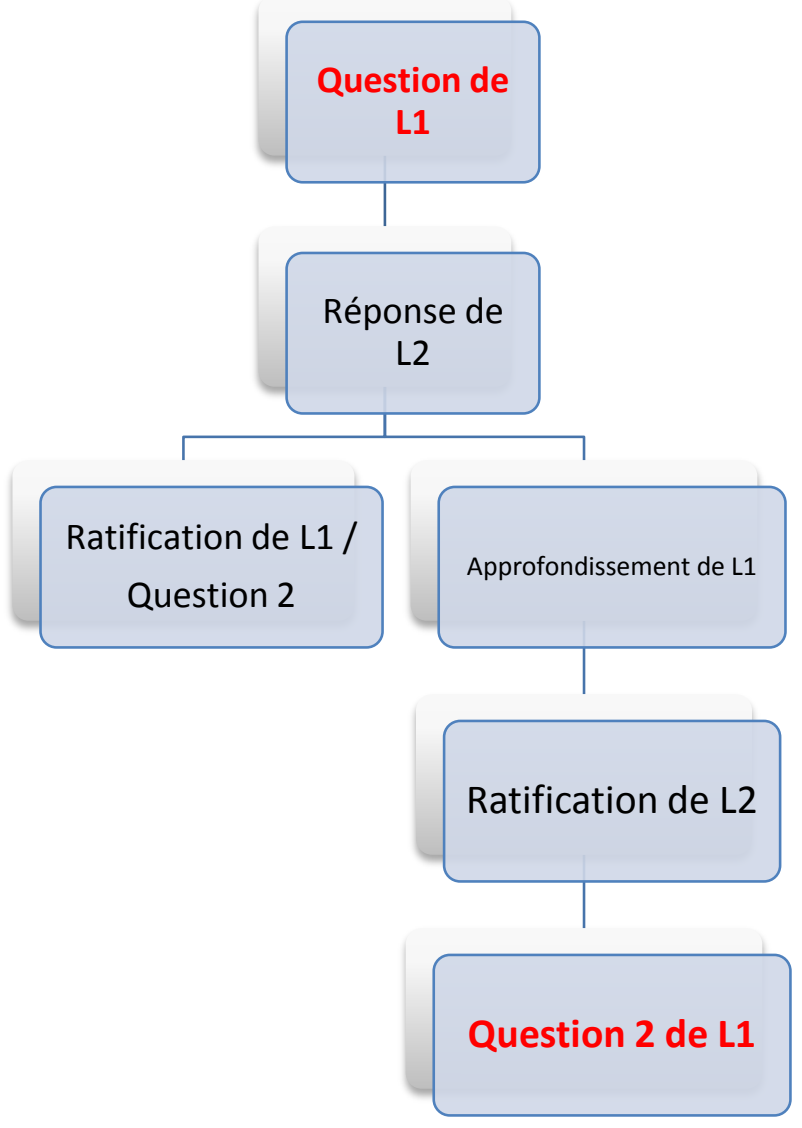

Lorsque le locuteur-conseiller tente d'approfondir la question la ratification de type : oui, non, ouais ou encore voilà que propose le locuteur-demandeur d'emploi - que nous appelons ratification fermée car elle coupe court à toute seconde tentative d'approfondissement du locuteur-conseiller - ne permet pas de prolonger l'échange sur ce thème. Dans l'exemple proposé ci-dessous, chaque réponse du locuteur-demandeur d'emploi est une reprise d'un ou 
des propos du locuteur-conseiller. L1 introduit deux thématiques «parler ou de remplir les papiers ». L2 construit sa réponse sur la reprise des propos de L1 : la difficulté «moi c'est difficile » et le fait de remplir des papiers «j'ai jamais rempli les papiers ». Pour approfondir, L1 reprend les propos de L2 « les papiers c'est difficile». L2 ratifie et conclut en réutilisant le « ouais » de L1.

\section{Exemple}

Question L1

L1 [...] est ce qu'il y a des situations où vous n'êtes pas à l'aise où c'est difficile pour vous de parler ou de remplir les papiers ou de choses comme ça

\section{Réponse L2}

L2 moi c'est difficile c'est remplir papiers j'ai jamais rempli les papiers

\section{Approfondissement $\mathrm{L} \mathbb{1}$ \\ L1 ouais les papiers c'est difficile}

\section{Ratification L2}

L2 ouais

[Sous corpus $01 /$ ENR $01 / 6^{\text {ème }}$ ]

Ce modèle de construction du discours est construit notamment sur l'utilisation de reprises diaphoniques de continuation.

\subsection{Analyse des phénomènes de reprise}

Par la répétition des propos du locuteur-demandeur d'emploi, le locuteur-conseiller tente de prolonger l'échange et ainsi obtenir une réponse satisfaisante. Nous rencontrons de nombreux exemples dans notre corpus illustrant différents types de reprises. Nous en avons extraits quelques exemples :

- La reprise en écho

\section{L2 ouais un peu de sport}

L1 un peu de sport vous faites du sport vous faites quoi

- Une reprise en écho personnalisée

L2 c'est pour + c'est pour le + chercher du boulot tout ça euh donner un coup de main euh 


\section{L1 oui alors vous donner un coup de main à quoi}

- Une reprise diaphonique réitérative suivie d'une question d'approfondissement

L2 ça ne me plait plus

L1 ça ne vous plait plus tellement alors est ce qu'il y a quelque chose qui vous plairait

Dans ces exemples, le locuteur qui produit la reprise essaie de donner à son interlocuteur l'occasion de poursuivre son discours. Sans ces reprises, le locuteur-demandeur d'emploi ne cherche pas ou peu à collaborer au discours.

La reprise peut également être un outil de vérification pour le locuteur-conseiller. Elle va alors lui servir à vérifier sa bonne compréhension des propos du locuteur-demandeur d'emploi faiblement qualifié. Ainsi après validation par le locuteur-demandeur d'emploi, le locuteurconseiller pourra poursuivre l'échange.

L1 [...] si je résume un petit peu hein l'emploi que vous aviez il n'existe plus

L2 ah ben oui

L1 hein il n'existe plus

L2 oui oui

Ainsi dans cet exemple, le locuteur-demandeur d'emploi n'est pas clair sur le statut de l'emploi qu'il occupait, L1 tente de reformuler ce qu'il a compris de son interlocuteur puis il vérifie sa compréhension, en répétant, avant de poursuivre son discours.

Le locuteur-demandeur d'emploi utilise lui aussi la reprise dans la construction de son propre discours. Cependant, ces reprises se manifestent généralement comme des stratégies de communication que nous pouvons qualifier d'astuces ou de tactiques pour gagner du temps ou encore, pour occuper le tour de paroles sans ajouter d'information nouvelle. Ces tactiques peuvent être analysées comme des traces discursives d'une insécurité langagière, nous en avons observées plusieurs dans notre corpus. Les exemples suivant illustrent ces phénomènes :

- L'auto-reprise sans apport d'information complémentaire

L1 mais avec un autre niveau $<$ avec d'autres compétences peut être maintenant

L2 voilà * avec la sous traitance euh>

L1 d'accord ok

L2 la sous traitance euh 


\section{L1 d'accord ok $[\ldots]$}

Dans ce premier exemple, le locuteur-demandeur d'emploi émet une proposition, le locuteurconseiller la ratifie. Dans le tour de parole suivant, le locuteur-demandeur d'emploi semble souhaiter poursuivre l'échange, mais en fait il ne fait que reprendre ses propos.

- La reprise diaphonique de ratification

L2 hum hum hum

L1 vous avez l'ASS

L2 ah *** 1'ASS oui je crois oui oui

L1 donc ça n'ira pas

L2 ça n'ira pas

Dans ce deuxième exemple, le locuteur-demandeur d'emploi utilise la reprise pour respecter les règles de construction et d'allocation des tours de parole c'est-à-dire prendre son tour de parole.

- La reprise identique pour répondre

L1 [...] euh vous avez eu des entretiens ces derniers temps +

L2 non

L1 pas ces derniers temps

L2 non je suis au chômage depuis même pas un mois là

L1 ah oui c'est tout récent

L2 oui c'est tout récent

Dans le troisième exemple, le locuteur-demandeur d'emploi s'appuie sur les propos du locuteur-conseiller pour construire sa propre réponse. Si les propos du locuteur-conseiller ont pu être repris dans le cadre d'interactions avec des locuteurs-demandeurs d'emploi qualifiés, ces reprises pouvaient être considérées comme des éléments discursifs d'introduction de leur propre discours.

- La reprise adaptée pour répondre

L1 ouais vous pouvez m'en dire un peu plus sur votre parcours professionnel

L2 euh vous voulez que je vous en dise moi personnellement [...]

Dans ce quatrième et dernier exemple, le locuteur-demandeur d'emploi tente une reprise personnalisée des propos du locuteur-conseiller. Si la syntaxe est respectée au niveau de l'utilisation du temps, la construction de cet énoncé est déviante : la reprise de «en »n'est pas appropriée. Une construction verbale satisfaisante aurait préféré que je vous en parle ou que je vous le dise. 
L'analyse des différents types de reprises rencontrées dans le discours du locuteur-demandeur d'emploi faiblement qualifié, et la fréquence d'utilisation de celles-ci pour participer aux interactions et pour construire collaborativement l'échange, nous montre la difficulté de ce public à se réapproprier le discours de l'autre pour construire son propre tour de parole. Cette construction n'est pas sans incidence sur l'intercompréhension et la poursuite de l'échange.

\section{Conclusion}

L'approche sociolinguistique des interactions permet de montrer l'importance du phénomène discursif de la reprise, outil fondamental dans la construction collaborative du discours et la négociation du sens de celui-ci. Les exemples que nous avons analysés montrent le rôle primordial du locuteur qualifié dans la construction collaborative du discours avec un locuteur-demandeur d'emploi faiblement qualifié. En effet, moteur de l'interaction verbale, l'implication du locuteur qualifié dans l'échange est la condition nécessaire pour dépasser les insécurités langagières du locuteur-demandeur d'emploi faiblement qualifié. Il est le garant d'une communication satisfaisante.

\section{Références bibliographiques}

ADAMI H., 2007. La « maîtrise de la langue » Des enjeux idéologiques aux enjeux scientifiques et pédagogiques. Diversité ville école intégration - $\mathrm{n}^{\circ} 151$ - décembre 2007

[En ligne] : http://www2.cndp.fr/archivage/valid/93997/93997-15508-19495.pdf

ANDRE V., 2010. Eléments de la construction collaborative du discours au sein des réunions de travail : La reprise et le couple oui, non. Pratiques. 148, p.199-222.

ANDRE V., 2014. L'énonciation conjointe : trace et ressource de la construction collaborative du discours. Actes du $4{ }^{\text {ème }}$ Congrès Mondial de Linguistique Française. p.1891-1904. [En ligne]

http://www.linguistiquefrancaise.org/articles/shsconf/pdf/2014/05/shsconf_cmlf14_01323.pdf

DE GAULMYN N.-M., 1987. Actes de reformulation et processus de reformulation. In BANGE GP., (Ed). L'analyse des interactions verbales. La dame de Caluire: une consultation. Berne : Peter Lang, pp.83-98.

DIVAY S., 2011. Une progressive rationalisation de l'encadrement des chômeurs. Langage et société. $2011 / 3-\mathrm{n}^{\circ} 137$ p. 91-111.

DUCROT O., 1980. Les mots du discours. Paris : Editions de Minuit.

FAERCH C., KASPER G., 1980. Stratégies de communication et marqueurs de stratégies. Encrages, p.17-24. 
GIACOMI A., DE HEREDIA C., 1986. Réussite et échecs dans la communication linguistique entre locuteurs francophones et locuteurs immigrés. Langages, n84, p.9-24. Paris : Larousse.

GOFFMAN E., 1981. Façon de parler. Paris : Minuit.

GRICE H.-P., 1979. Logique et conversation. Communications, 30, p.57-72.

GÜLICH E., KOTSCHI T., 1987. Les actes de reformulation dans la consultation La Dame de Caluire, in BANGE P., (Ed).1987. L'analyse des interactions verbales. La dame de Caluire : une consultation. Berne : Peter Lang, p.15-81.

HYMES D., 1972. Models of the interaction of language and social life In GUMPERZ J., HYMES D. (Eds), Directions in Sociolinguistics. The Ethnography of Communication, Oxford : Basil Blackwell, p.35-71.

KERBRAT-ORECCHIONI C., 1996. La conversation. Memo Seuil.

KERBRAT-ORECCHIONI C., 2000. L'analyse des interactions verbales: la notion de négociation conversationnelle : défense et illustration. Laliés, 20 p.63-141.

KERBRAT-ORECCHIONI C., 2005. Le discours en interaction. Paris : Armand Collin.

ROULET E., 1985. L'articulation du discours en français contemporain. Berne : Lang.

SACKS H., SCHEGLOFF E., JEFFERSON G., 1974. A simplest systematics for the organization of turn-taking in conversation. Language, volume 50, p.696-735.

SCHEGLOFF E.A., SACKS H., 1973. Opening up closings. Semiotica.

SCHEGLOFF E.A., 1982. Discourse as an international achievement. Some uses of "Uh huh" and other things that come between sentences. p.71 à 93 In Analyzing discourse. Text and talk. TANNEN D., (Ed.) Washington Georgetown University Press.

SCHEGLOFF E.A., 1987. Some sources of misunderstanding in talk-ininteraction. Linguistics, ${ }^{\circ} 25$, p.201-218

VION R., 1992. La communication verbale. Paris : Hachette.

VION R., 2005. Reprises et modes d'implication énonciative. Communication présentée au colloque Répétitions, Reprises et Reformulation. Quels usages dans les interactions verbales ? Paris, ler et 2 avril 2005.

\section{Convention de transcription}

Les conventions de transcription sont celles du projet TCOF (http://www.cnrtl.fr/corpus/tcof/).

$\{\ldots\} \quad$ Eléments métalinguistiques ou commentaires (rires, bruits, etc.) 


$\begin{array}{ll}\$ \$ \$ & \text { Coupure de l'enregistrement } \\ <\ldots> & \text { Chevauchements de locuteurs } \\ + & \text { Pauses } \\ / / / & \text { Pause très longue ou silence } \\ \ldots- & \text { Amorces } \\ * & \text { Syllabe incompréhensible } \\ * * * & \text { Suite de syllabes incompréhensibles } \\ * \mathrm{P}^{*} & \text { Anomysation d'un patronyme } \\ * \mathrm{~T}^{*} & \text { Anomysation d'un toponyme } \\ * \mathrm{~S}^{*} & \text { Anomysation d'un nom de société }\end{array}$

